\title{
Inhibition of formyl peptide receptor 1 activity suppresses tumorigenicity in vivo and attenuates the invasion and migration of lung adenocarcinoma cells under hypoxic conditions in vitro
}

\author{
Bo Huang ${ }^{1}$, Hongrong Guo ${ }^{1}$, Jie Ding ${ }^{2}$, Jun $\mathrm{Li}^{2}$, Hongjuan Wang ${ }^{1}$, Jianqun $\mathrm{Xu}^{1}$, Quan Zheng ${ }^{1}$, \\ Lijun Zhou ${ }^{1}$, Qin Dai ${ }^{1}$ \\ ${ }^{1}$ Department of Respiration, ${ }^{2}$ Department of Nephrology, Wuhan Third Hospital (Tongren Hospital of Wuhan University), Wuhan, China \\ Contributions: (I) Conception and design: B Huang, H Guo; (II) Administrative support: J Ding, J Li; (III) Provision of study materials or patients: H \\ Wang, J Xu; (IV) Collection and assembly of data: Q Zheng, L Zhou, Q Dai; (V) Data analysis and interpretation: B Huang, H Guo; (VI) Manuscript \\ writing: All authors; (VII) Final approval of manuscript: All authors. \\ Correspondence to: Bo Huang. Department of Respiration, Wuhan Third Hospital (Tongren Hospital of Wuhan University), No. 216, Guanshan \\ Avenue, Hongshan District, Wuhan, China. Email: doctorhuang82@126.com.
}

\begin{abstract}
Background: Tumor hypoxia has been widely reported to promote metastasis. However, the molecular mechanisms underlying metastasis-associated hypoxia remain unclear. Formyl peptide receptor 1 (FPR1) has been reported to be highly expressed under hypoxic conditions. This study aimed to explore the role of FPR1 in tumor cells under hypoxic conditions.

Methods: The expressions of FPR1 and hypoxia-inducible factor $1 \alpha(\mathrm{HIF}-1 \alpha)$ in A549 cells under hypoxic conditions were detected using western blot. The expression of FPR1 in A549 cells under hypoxic conditions was suppressed using the FPR1 antagonist Boc2. Wound-healing and Transwell assays were performed to investigate the migration and invasion of cells. Furthermore, the tumorigenicity of A549 cells was evaluated by constructing a hypoxic mouse model of lung adenocarcinoma. The expression levels of HIF-1 $\alpha$ and FPR1 in tumors were measured by real-time polymerase chain reaction (PCR) and western blot.

Results: The expression levels of FPR 1 and HIF-1 $\alpha$ increased in a time-dependent manner after exposure to hypoxic conditions. Wound-healing and Transwell assays showed that hypoxia promoted the migration and invasion abilities of A549 cells, whereas downregulation of FPR1 blocked the effects of hypoxia on A549 cells. Our in vivo results demonstrated that the tumor volumes and weights of mice exposed to hypoxic conditions were significantly higher than those of untreated mice. Furthermore, the downregulation of FPR1 blocked the effects of hypoxia in the mice. Meanwhile, the expressions of HIF-1 $\alpha$ and FPR1 at the protein and mRNA levels were increased after hypoxic exposure, whereas FPR1 antagonist Boc2 suppressed the effect of hypoxia on the expression of FPR1.

Conclusions: Our results suggest that FPR1 could be a therapeutic target for suppressing the invasion and tumorigenicity of lung adenocarcinoma cells.
\end{abstract}

Keywords: Formyl peptide receptor 1 (FPR1); migration; invasion; tumorigenicity; hypoxia

Submitted Jul 21, 2020. Accepted for publication Sep 09, 2020.

doi: $10.21037 / \mathrm{atm}-20-5864$

View this article at: http://dx.doi.org/10.21037/atm-20-5864 


\section{Introduction}

Lung cancer is responsible for more than 1 million deaths annually, making it the leading cause of cancer-related mortality worldwide. Adenocarcinoma is the most common histological type of lung cancer (1). In China, lung cancer is ranked first for tumor-related morbidity and mortality in men, and second and first for tumor-related morbidity and mortality, respectively, in women. Over the past decade, significant progress has been made in the diagnosis and treatment of lung cancer, including screening, diagnostic examination, surgery, radiotherapy, and chemotherapy. Biological targeted therapies have significantly improved the prognoses of patients whose tumors harbor somatically activated oncogenes, such as translocated anaplastic lymphoma kinase (ALK) $(2,3)$ or mutant epidermal growth factor receptor 1 (EGFR1) (4). However, some lung adenocarcinomas lack an identifiable driver oncogene or harbor mutations in KRAS (Kirsten rat sarcoma viral oncogene), meaning they are not sensitive to current biological treatments (1). Characterizing the signaling pathways that control cell survival, proliferation, and invasion in different tumors is pivotal to the development of more effective cancer treatments.

Hypoxia has been put forward as a decisive marker of the biological behavior of malignant solid tumors. Some experimental studies have demonstrated that tumor hypoxia leads to increased risk of local recurrence and distant metastasis, as well as radiotherapy and chemotherapy resistance $(5,6)$. Investigators recently confirmed that hypoxia induces tumor metastasis, with a strong correlation existing between tumor hypoxia, metastasis, and poor prognosis in cancer patients $(7,8)$.

Formyl peptide receptor 1 (FPR1) is a G proteincoupled receptor that was initially identified in phagocytic leukocytes. Subsequent studies found that FFR1 could mediate cell chemotaxis and be activated by $\mathrm{N}$-formylmethionyl-leucyl-phenylalanine (9). FFR1 is involved in the pathophysiological processes in tumors, including inflammation (10-13), glioblastoma progression $(14,15)$, migration (16), invasion (17), and tumorigenicity $(9,18)$. Cai et al. showed that hypoxia induced the expression of FPR1 in cancer tissues (19). The involvement of FPR1 in tumorigenesis is seemingly correlated with the specific conditions (specific tumor, stage). For instance, an association has been documented between high expression of FPR1 and poor survival in gastric cancer and advanced disease (20). However, FPR1 has also been reported to inhibit angiogenesis in gastric cancer (21).
In the present study, we examined changes in FPR1 expression at the protein and mRNA levels by establishing a tumor hypoxia model in vitro and in vivo. Additionally, we investigated the effects of FPR1 on the growth, migration, and invasion of tumor cells after hypoxic exposure. We found that inhibition of FPR1 activity suppressed the migration and invasion abilities of A549 cells, and also suppressed tumor growth following hypoxia in a nude mice tumor model constructed with stably transfected A549 cells. We present the following article in accordance with the ARRIVE reporting checklist (available at http://dx.doi. org/10.21037/atm-20-5864).

\section{Methods}

\section{Cell culture and treatment}

A549 cells (Kunming Institute of Zoology, Kunming, China) were cultured in Roswell Park Memorial Institute (RPMI) 1640 medium (Invitrogen, USA) supplemented with $10 \%$ fetal bovine serum and $1 \%$ penicillin-streptomycin. Cells were incubated at $37{ }^{\circ} \mathrm{C}$ in a humid atmosphere containing $5 \% \mathrm{CO}_{2}$ and $5-7 \% \mathrm{O}_{2}$ (hypoxic conditions) for $0 \mathrm{~h}(21 \%$ $\left.\mathrm{O}_{2}\right), 12,24$, or $48 \mathrm{~h}$. After incubation, the expression levels of hypoxia-inducible factor $1 \alpha(\mathrm{HIF}-1 \alpha)$ and FPR1 in the cells were detected by western blot. Boc2, an antagonist of FPR1, was supplied by Shanghai Apeptide Co., Ltd. (Shanghai, China). A549 cells grown to $90 \%$ confluence and pretreated with Boc2 $(10 \mathrm{mM})$ for $30 \mathrm{~min}$ were subsequently cultured in non-hypoxic (the Boc2 group) or hypoxic (the hypoxia + Boc2 group) conditions for $24 \mathrm{~h}$. Cells cultured for $24 \mathrm{~h}$ without Boc2 in a humid atmosphere containing $5 \% \mathrm{CO}_{2}$ in non-hypoxic or hypoxic conditions served as the control and hypoxia groups, respectively.

\section{Cell viability}

A549 cells were seeded overnight in a 96-well plate at a concentration of $2 \times 10^{4}$ cells/well, and then treated with or without Boc2 $(10 \mathrm{mM})$ for $30 \mathrm{~min}$. Following that, the cells were cultured in non-hypoxic or hypoxic conditions for $24 \mathrm{~h}$. The cell viability was measured by MTT assay according to the manufacturer's instructions. The results were expressed as percentages, with the control group taken as $100 \%$.

\section{Wound-healing assay}

To examine cell migration ability, A549 cells were seeded in 6-well plates with RPMI-1640 medium containing 10\% 
fetal bovine serum and grown to $90 \%$ confluence. The cells were starved by changing the medium to serum-free RPMI-1640 and then subjected to different treatments for $24 \mathrm{~h}$. The cells were scratched with a $100 \mu \mathrm{L}$ plastic pipette tip and then washed with phosphate-buffered saline (PBS) before treatment. The wound was then photographed using an inverted phase contrast microscope (Nikon; magnification: $10 \times$ ), and the migration distances were measured 24 hours after treatment.

\section{Cell invasion assay}

The invasive capacity of A549 cells after different treatments were detected in $8.0-\mu \mathrm{m}$ pore size Transwell 24 -insert plate chambers. Cells $\left(5 \times 10^{4} /\right.$ well $)$ were seeded in the upper chamber with $200 \mu \mathrm{L}$ serum-free medium, while complete culture medium with a high concentration of serum was placed in the lower chamber to trap invading cells. After incubation for $24 \mathrm{~h}$, the cells in the upper chamber were removed. The cells that had penetrated the Matrigel-coated membranes and migrated into the lower chamber were stained with crystal violet $(0.1 \%)$ and photographed. The invasion activity in each sample was quantified by counting the crystal violet-stained cells.

\section{Hypoxic mouse model of lung adenocarcinoma}

Nude BALB/C mice (weight, 21-23 g) were supplied by Chongqing Medical University (Chongqing, China). The mice were individually housed in plastic cages (cage size, $20 \mathrm{~cm} \times 15 \mathrm{~cm} \times 15 \mathrm{~cm}$ ) under a 12-h light-dark cycle. The mice had free access to food and water in their cages, but not during experimental procedures.

The mouse hypoxia model was established by subcutaneously injecting the mice with $2 \times 10^{4}$ A549 cells $(500 \mu \mathrm{L})$ according to the previous study (22). Briefly, unanesthetized, the mice were placed in 6-liter incubator chambers, where they were exposed to a continuous flow of a humidified gas mixture to induce hypoxia. The mixed gas intervention involved exposure to $5-7 \% \mathrm{O}_{2}$ and $95-93 \% \mathrm{~N}_{2}$, for $2 \mathrm{~h}$ once a day, 7 days a week. During the treatments, the flow rate was monitored and maintained at 3-6 liters/min, and the temperature was kept constant at room temperature. During the phases of hypoxia treatment, mice were treated with Boc2 $(50 \mu \mathrm{g} / \mathrm{kg}$, the hypoxia + Boc2 group) or saline (the hypoxia group) once a day. Mice in the Boc2 and control groups were placed in the same chambers and exposed to a continuous mixed flow of humidified room air with saline and Boc2 treatment, respectively. Tumor growth in the mice was observed once a day, and the tumor volumes were measured once every 2 days. After 2 weeks of hypoxia exposure, the mice were sacrificed. Only one tumor was observed in the animals in our study. The maximum diameter of a single tumor was $1.5 \mathrm{~cm}$. This subcutaneous tumor was rapidly dissected 7 days after the injection, and its weight and volume were measured. The volume of the tumor was calculated using the following formula: $\mathrm{V}=$ $\pi \mathrm{abc/6}$ (a, tumor length; b, tumor width; c, tumor height). Pathological changes in the tumor were examined using. All procedures involved in the animal experiments in this study were performed in line with the National Institutes of Health Guide for the Care and Use of Laboratory Animals. The study was approved by the Ethics Committee of Wuhan Third Hospital.

\section{Real-time polymerase chain reaction (PCR)}

Real-time PCR assay was carried out to detect the mRNA expression of HIF- $1 \alpha$ and FPR1 in the tumor tissue. The Premix Ex Taq kit (Takara, Japan) and a 7300 real-time PCR system (Life Technologies, USA) were used to perform of real-time PCR, according to the manufacturer's instructions. The thermocycler conditions for PCR were set as follows: $25^{\circ} \mathrm{C}$ for $5 \mathrm{~min}, 42^{\circ} \mathrm{C}$ for $60 \mathrm{~min}, 75^{\circ} \mathrm{C}$ for $5 \mathrm{~min}$, and $4{ }^{\circ} \mathrm{C}$ for 5 min (23). The mRNA expression levels of HIF-1 $\alpha$ and FPR1 were normalized to the endogenous expression of $\beta$-actin. The relative mRNA level was calculated using the $2^{-\Delta \Delta C t}$ method (24). The real-time PCR primer used was: mouse $\beta$-actin F: 5 ' -CAGTTCGCCATGGATGAC GAT3', R: 5'-ATCTGGGTCATCTTTTCACGGTTG-3'; mouse HIF- $\alpha$ F: 5'-CCCAT CCATGTGACCATGAGG-3, R: 5 ' - TCAGCACCAAGCACGTCATAGG-3 '; mouse FPR 1 F: 5'-GCTACTAACTTCTCT CTATTAAAGCAAGCAGGAGACGTGGAAGA AAACC CAGGTCCTATGGAGACAAATTCCTCTCTCCC-3', R: 5' -GCGGAGGCC ACGCGTCTACTTTGCCTGTA ACTCCACC-3'.

\section{Western blot}

Western blot was performed to evaluate the protein expressions of HIF- $1 \alpha$ and FPR1 in A549 cells exposed to a hypoxic atmosphere for different lengths of time ( 0 , 12,24 , and $48 \mathrm{~h}$ ), as well as in tumor tissues. Cells were washed three times in ice-cold PBS. Cell lysis buffer (R0010, SOLARBIO, Beijing, China) was subsequently added, and 
A
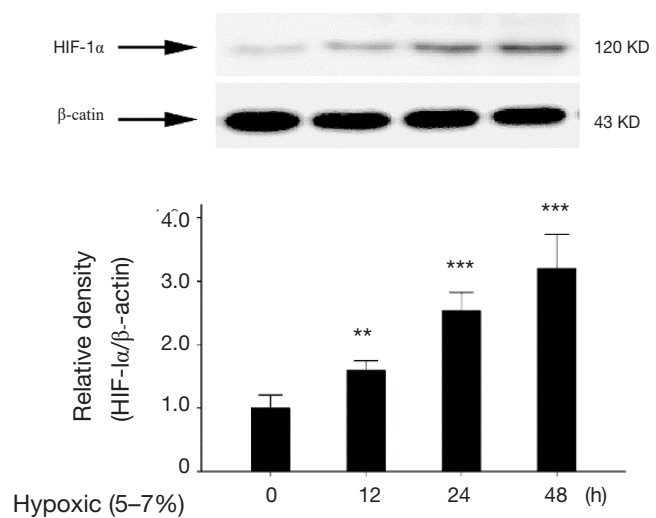

B
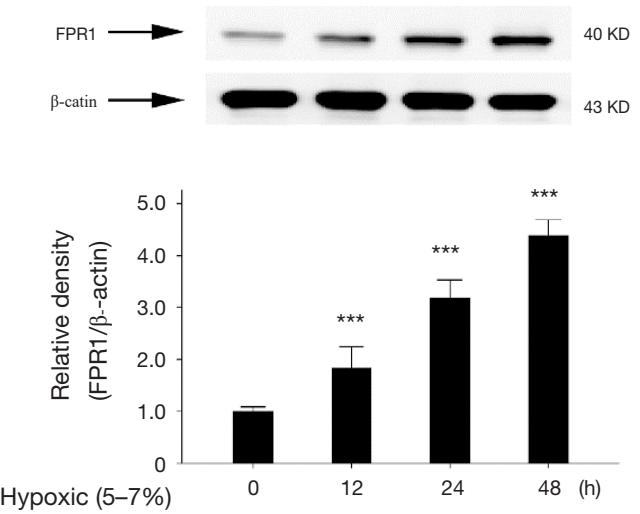

Figure 1 The expression levels of HIF-1 $\alpha$ and FPR1 in A549 cells under hypoxic conditions. Hypoxia induced the expression of (A) HIF-1 $\alpha$ and (B) FPR1 after treatment for 12, 24, and 48 h. Asterisks indicate statistical significance $(* *, P<0.01$, ***, $\mathrm{P}<0.001)$. Data are presented as the means \pm SD $(n=3)$. HIF-1 $\alpha$, hypoxia-inducible factor $1 \alpha$; FPR1, formyl peptide receptor 1; SD, standard deviation.

the cells were incubated on ice for $1 \mathrm{~h}$ for protein extraction. The obtained tumor tissues were homogenized in RIPA lysis buffer (Bioswamp, Wuhan, China) and centrifuged at $12,000 \mathrm{~g}$ for $15 \mathrm{~min}$. Protein concentration was measured using the BCA Protein Assay Kit (Beyotime Biotechnology, China) in line with the manufacturer's instruction. Equal amounts of protein $(20 \mu \mathrm{g})$ were separated with $12 \%$ sodium dodecyl sulfate-polyacrylamide gel electrophoresis (SDS-PAGE) and then transferred to a polyvinylidene difluoride (PVDF) membrane (Millipore Corporation, Billerica, MA, USA). The membrane was blocked with $10 \%$ skimmed milk (in Tris-buffered saline (TBS), $\mathrm{PH}$ 7.2 , containing $0.1 \%$ Tween-20) for $2 \mathrm{~h}$ and incubated at $4{ }^{\circ} \mathrm{C}$ overnight with primary antibody (working dilutions of antibodies: HIF-1 $\alpha$ (\#36169, CST, MA, USA): 1:1,000; FPR1 (ab113531, Abcam, Cambridge, UK): 1:1,000; and $\beta$-actin 20536-1-AP, Proteintech, MI, USA): 1:400. Then, the membrane was incubated with peroxidase-conjugated anti-rabbit secondary antibodies (1:5,000, sc-20026, KPL, Gaithersburg, MD, USA) for $1 \mathrm{~h}$ at room temperature and visualized by chemiluminescence (Milipore Corporation, USA). ImageJ software (NIH, USA) was used to perform densitometry analysis.

\section{Statistical analysis}

Data were analyzed using SPSS 23.0 software (Illinois, USA). Continuous data were expressed as mean \pm standard deviation (SD). Student's $t$-test was used to compare the data between two groups from the cellular experiments.
Comparisons between multiple groups were performed by one-way analysis of variance (ANOVA), followed by a Bonferroni post-hoc test. $\mathrm{P}<0.05$ was considered to show statistical significance.

\section{Results}

\section{Hypoxia induced the expressions of HIF-1 $\alpha$ and FPR 1 in A549 cells}

As the main regulator of gene expression induced by low oxygen levels, HIF- $1 \alpha$ is able to respond to a wide range of environmental oxygen concentrations (25). We examined the expressions of HIF- $1 \alpha$ and FPR1 in A549 cells after hypoxic exposure for $0,12,24$, and $48 \mathrm{~h}$. The results showed that the expression of HIF- $1 \alpha$ increased in a time-dependent manner (Figure 1A). Similarly, the expression of FPR1 also increased in a time-dependent manner (Figure 1B).

\section{Inbibition of FPR1 activity suppressed the migration and invasion abilities of A549 cells under bypoxic conditions}

To evaluate the biological effect of FPR1 on the migration and invasion abilities of lung adenocarcinoma cells, Boc2, a specific antagonist of FPR1, was used to inhibit FPR1 activity in A549 cells. A549 cells were pretreated with Boc2 for $30 \mathrm{~min}$, and then exposed to hypoxic conditions for $24 \mathrm{~h}$. As shown in Figure $2 A$, hypoxia significantly increased the viability of A549 cells, while Boc2 partially inhibited the 
A

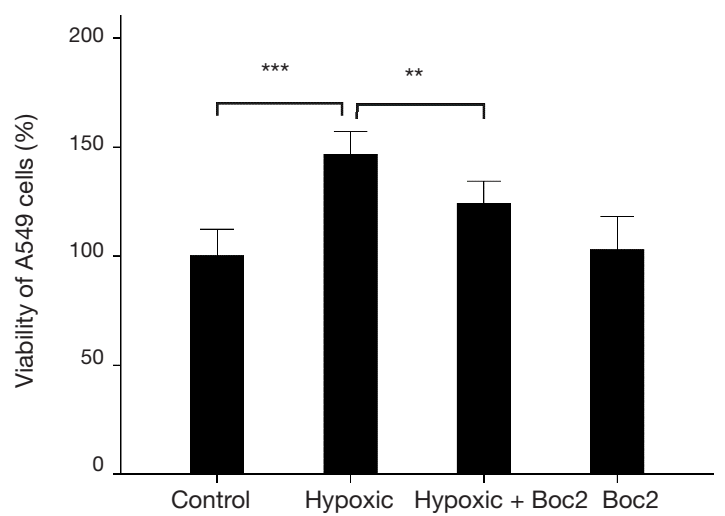

B

Control

Hypoxic

Hypoxic + Boc2

Boc2
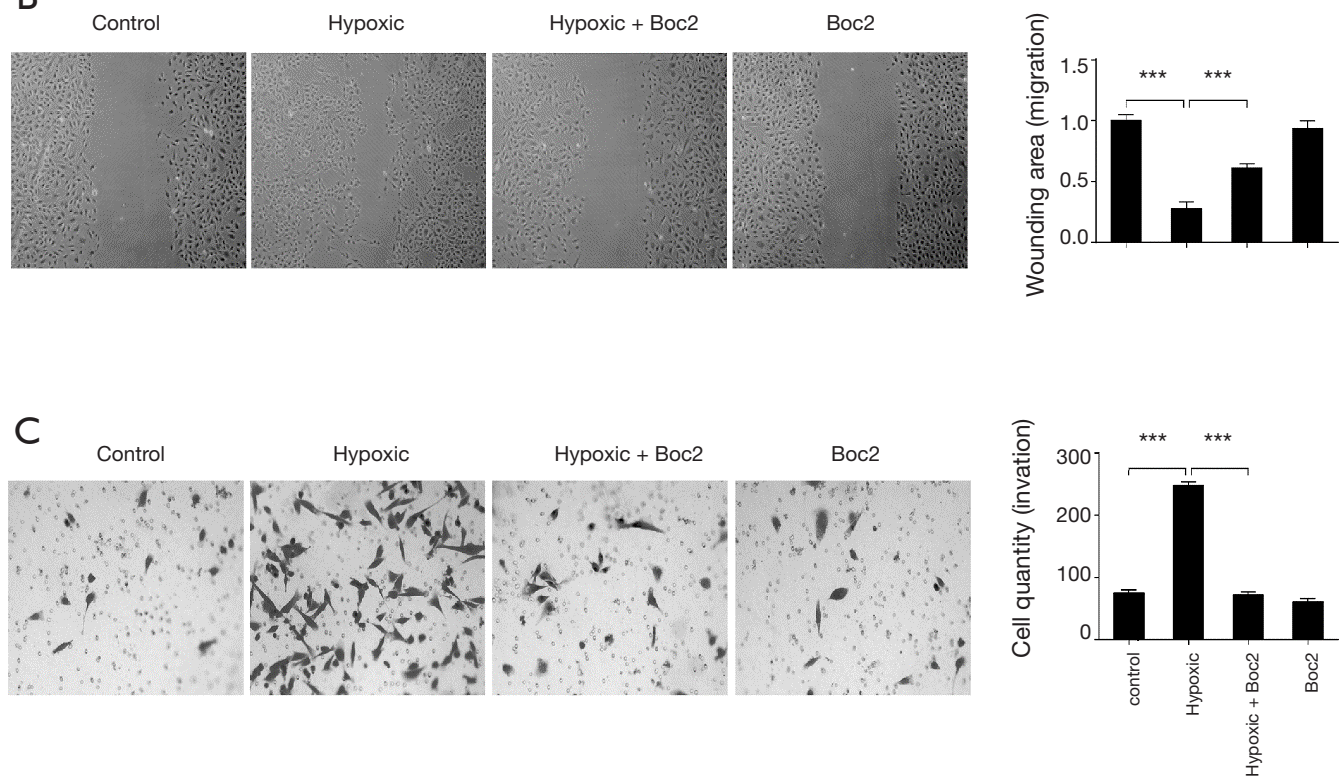

Figure 2 The migration and invasion of A549 cells under hypoxic conditions. Inhibition of FPR1 activity suppressed the (A) viability, (B) migration ( $\times 100)$, and (C) invasion of A549 cells induced by hypoxia $(\times 200)$. Asterisks indicate statistical significance ${ }^{* *}, \mathrm{P}<0.01,{ }^{* * *}$, $\mathrm{P}<0.001)$. Data are presented as the means $\pm \mathrm{SD}(\mathrm{n}=3)$. FPR1, formyl peptide receptor 1 ; SD, standard deviation.

hypoxia-induced changes. Cells viability in the Boc2 group was similar to that in the control group. Wound-healing assay showed that hypoxia enhanced the migration of A549 cells in the hypoxia group compared with that in the control group, whereas the inhibition of FPR1 activity suppressed wound healing in the hypoxia + Boc2 group compared with in the hypoxia group (Figure 2B). These results indicate that hypoxia promotes tumor cell migration, while the inhibition of FPR1 suppresses the migration of A549 cells induced by hypoxia. The most damaging malignant characteristic of lung adenocarcinoma is the potential for invasion and metastases induced by hypoxia. Here, Transwell assay was conducted to examine whether FPR1 also affects cell invasion. The number of cells in the lower chamber after exposure to hypoxia was significantly greater than that in the control group, whereas the inhibition of FPR1 activity via the administration of Boc2 suppressed the invasion ability of A549 cells (Figure 2C). These results indicated 
A

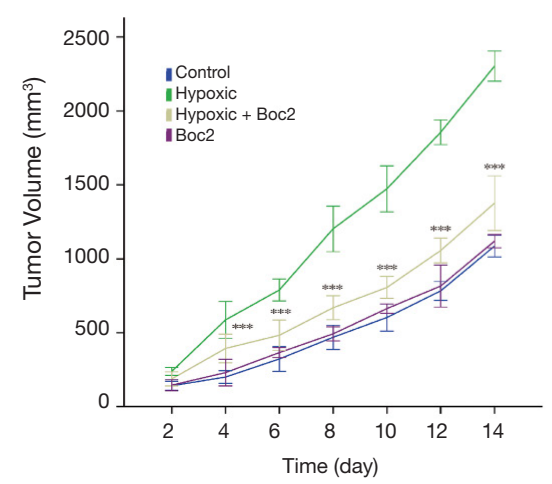

C

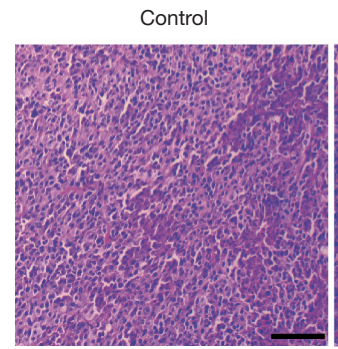

Hypoxic

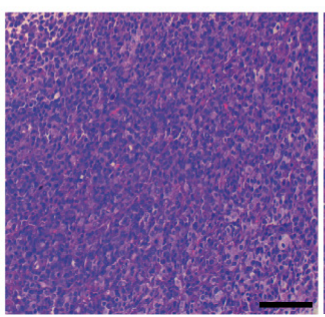

B

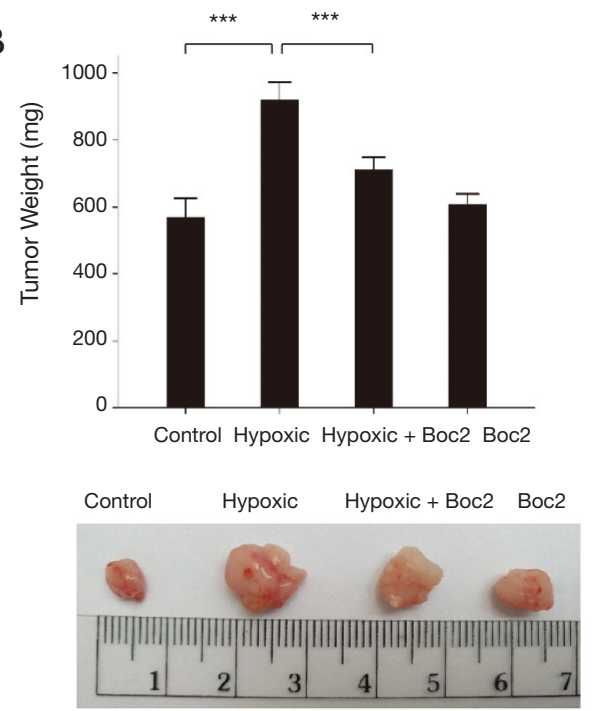

Figure 3 Tumor growth in nude mice under hypoxic condition. Inhibition of FPR1 activity suppressed the hypoxia-induced increase in (A) tumor volume and (B) tumor weight. (C) Inhibition of FPR1 activity suppressed the proliferation of tumor cells. Asterisks indicate statistical significance ${ }^{* * *}, \mathrm{P}<0.001$ ). Data are presented as the mean \pm SD (experiments, $\mathrm{n}=3$ ). Scale bar $=100 \mu \mathrm{m}$. FPR1, formyl peptide receptor 1 ; $\mathrm{SD}$, standard deviation.

that inhibition of FPR1 activity suppressed the migration and invasion of A549 cells under hypoxic conditions.

\section{Inbibition of FPR1 activity suppressed tumor growth in nude mice under hypoxia}

To investigate the effect of FPR 1 on the lung adenocarcinoma tumor growth in vivo, A549 cells $\left(2 \times 10^{4}\right)$ were injected into right forelimbs of 7-week-old nude mice to establish a tumor xenograft model. After the injection, the mice were exposed to hypoxia for $2 \mathrm{~h}, 7$ days a week for 2 weeks; during this period, mice were also treated with Boc2 $(50 \mu \mathrm{g} / \mathrm{kg})$ or saline once a day. The tumor size was first measured at 2 days after the injection. After 14 days of observation, the tumors were removed from the mice, and the tumor volumes and weights were measured. The hypoxia group had larger and heavier tumors than the control group; however, the inhibition of FPR1 activity resulted in a significant reduction in tumor growth in the hypoxia + Boc2 group compared with in the hypoxia group (Figure $3 A, B)$. Figure $3 C$ shows the pathological changes in the tumors, revealing that the proliferation of tumor cells was significantly increased by hypoxia, but this was attenuated by Boc2. Meanwhile, compared to the control group, the inhibition of FPR1 activity under normoxic conditions had little effect on the proliferation of the tumor cells. These results indicated that inhibition of FPR1 activity not only inhibited the migration and invasion of 

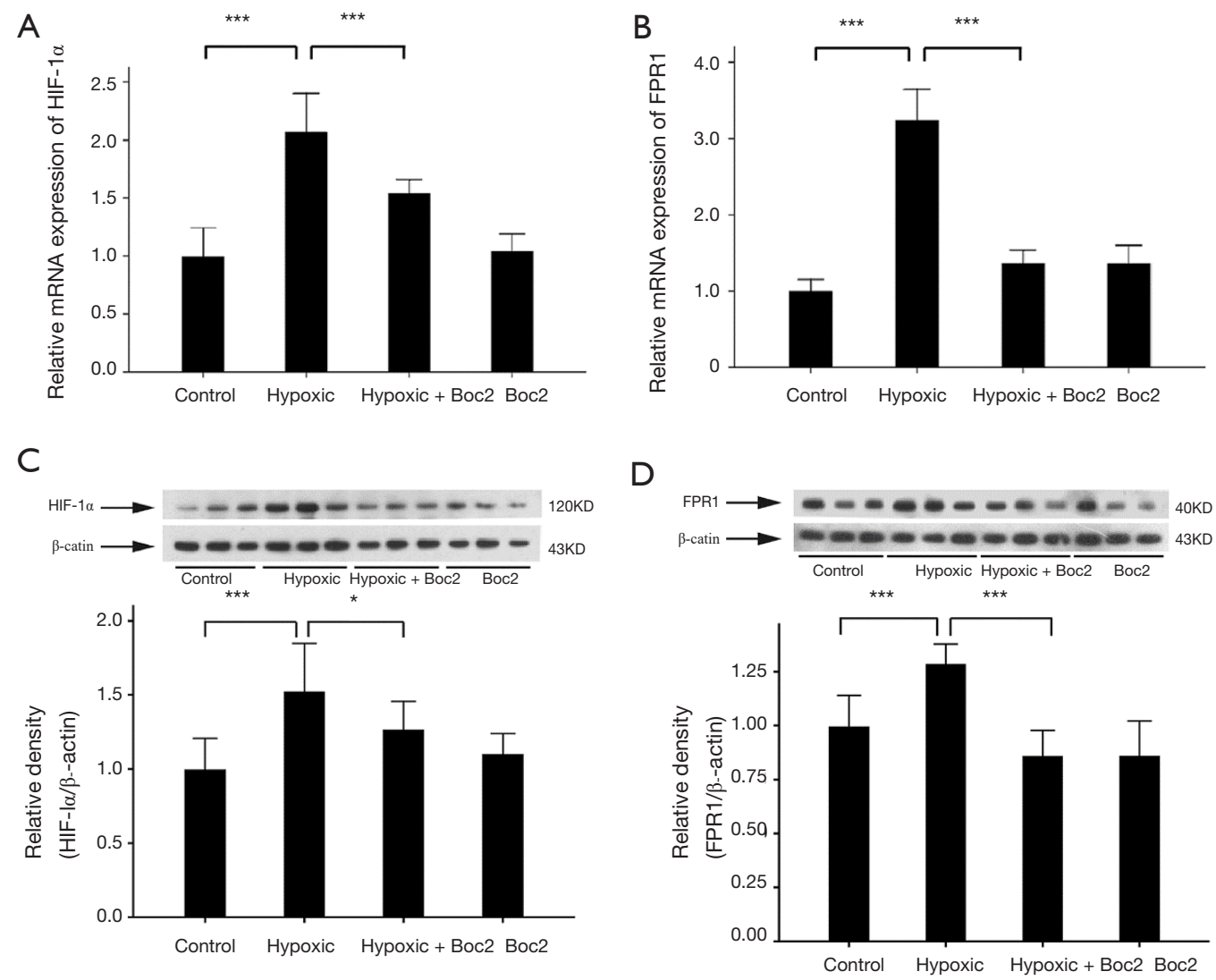

Figure 4 The expression levels of HIF-1 $\alpha$ and FPR1 in the tumor tissues of nude mice. The expression levels of (A) HIF-1 $\alpha$ and (B) FRP1 mRNA in the tumor tissues. The protein expression levels of (C) HIF-1 $\alpha$ and FRP1 (D) in the tumor tissues. Asterisks indicate statistical significance $\left.{ }^{*}, \mathrm{P}<0.05,{ }^{* * *}, \mathrm{P}<0.001\right)$. Data are presented as the mean $\pm \mathrm{SD}$ (experiments, $\mathrm{n}=3$ ). HIF-1 $\alpha$, hypoxia-inducible factor $1 \alpha$; FPR1, formyl peptide receptor $1 ; \mathrm{SD}$, standard deviation.

lung adenocarcinoma cells but also significantly reduced hypoxia-induced tumor growth.

\section{Inhibition of FPR1 activity suppressed FPR1 expression in the tumors of nude mice under hypoxic conditions}

After the tumor volumes and weights were measured, we further examined the expression levels of HIF- $1 \alpha$ and FPR1 in the tumors. As shown in Figure 4, the expression of HIF$1 \alpha$ and FPR 1 at both protein and mRNA level increased after hypoxia treatment, whereas inhibition of FPR1 activity suppressed the effect of hypoxia on the expression of FPR1.

\section{Discussion}

This study investigated the role of FPR1 in the migration and invasion of lung adenocarcinoma cells, as well as in the growth of lung adenocarcinoma tumors under hypoxic conditions. Our results demonstrated that the expression level of FPR1 was increased significantly following hypoxia exposure, while the inhibition of FPR1 activity could suppress the migration and invasion abilities of tumor cells, as well as the growth of hypoxia-induced tumors in a mouse model. These results present FPR1 as a potential biomarker and possible therapeutic target in the treatment of lung adenocarcinoma.

HIF-1 $\alpha$, which is widely recognized to be a master regulator of the transcriptional response to low oxygen levels, responds to a wide range of environmental oxygen conditions (25). HIF- $1 \alpha$ can regulate the expression of more than 100 genes and plays a key role in the body's physiological and pathological processes. Based on previous 
studies, we first examined the expression of HIF-1 $\alpha$ in A549 cells and mice after hypoxia exposure, which revealed a higher expression of HIF- $1 \alpha$ under hypoxic conditions than under normoxic conditions, thus confirming the successful establishment of the hypoxia model in A549 cells and mice (26).

A number of studies have suggested that tumor cell migration and invasion are strongly influenced by microenvironmental factors, including a lack of nutrients, changes in oxygen availability, and the composition of the extracellular matrix (27-29). In particular, low oxygen tension, or hypoxia, is associated with metastasis through its stimulation of events leading to angiogenesis and tissue invasion (30), as well as with poor patient prognosis (31). However, the molecular mechanisms underpinning the relationship between hypoxia and metastasis are poorly understood. Hypoxia has been shown to increase the expression of FPR1 (19). Previous studies have reported FPR1 to be involved in the processes of migration, invasion, and tumorigenicity in various tumor cells. For instance, formylmethionyl-leucyl-phenylalanine, a specific agonist of FPR1, was found to enhance the migration of BV-2 cells in a dose-dependent manner, whereas Boc2 was observed to inhibit these effects (32). Moreover, FPR1 was reported to enhance the invasiveness of ovarian cancer cells through its interaction with leucine leucine-37 (17). Prevete et al. showed that the activation of FRP1 induced proliferation, migration, and resistance to apoptosis in gastric cancer cells; however, these changes could be reverted by blocking FRP1 (21). Moreover, FPR1 mediates the tumorigenicity of human hepatocellular carcinoma cells and neuroblastoma cells $(9,18)$. Thus, we hypothesize that the regulation of FPR1 activity may affect metastasis and tumor growth induced by hypoxia.

This study found that the expression of FPR1 increased in a time-dependent manner in A549 cells after hypoxia exposure, while the inhibition of FPR1 activity suppressed the hypoxia-induced migration and invasion of A549 cells. These findings are consistent with those of the previous study, as mentioned above $(9,18,21)$. The results of our in vivo experiment demonstrate that the volumes and weights of tumors in mice exposed to hypoxia were significantly higher than those of tumors in untreated mice, whereas the inhibition of FPR1 blocked the effects of hypoxia on tumor volumes and weights in mice. Meanwhile, FPR1 expression was increased at both the protein and mRNA levels following hypoxia exposure, while inhibition of FPR1 activity suppressed the effect of hypoxia on the expression of FPR1. Taken together, our results suggest that inhibition of FPR1 activity not only suppresses the migration and invasion of A549 cells, but also suppresses tumor growth.

\section{Conclusions}

In summary, we showed that hypoxia induced the expression of FPR1, enhanced tumor cell migration and invasion, and promoted tumor growth. The inhibition of FPR1 suppressed the migration and invasion of tumor cells in vitro and tumor growth in mice in vivo. Therefore, the pharmacological inhibition of FPR 1 may be a potential therapeutic strategy for the treatment of lung adenocarcinoma. Further studies are needed to determine whether siRNA targeting of FPR1 has similar effects in the regulation of tumor growth and invasion in lung adenocarcinoma under hypoxic conditions.

\section{Acknowledgments}

Funding: This study was supported by the Project of Health and Family Planning Commission of Wuhan Municipality (WX16D11) and the Wuhan Young and Middle-aged Medical Backbone Talent Training Project (Notice of Wuhan Municipal Health Commission (2019, No. 87).

\section{Footnote}

Reporting Checklist: The authors have completed the ARRIVE reporting checklist. Available at http://dx.doi.org/10.21037/ atm-20-5864

Data Sharing Statement: Available at http://dx.doi. org/10.21037/atm-20-5864

Conflicts of Interest: All authors have completed the ICMJE uniform disclosure form (available at http://dx.doi. org/10.21037/atm-20-5864). The authors have no conflicts of interest to declare.

Ethical Statement: The authors are accountable for all aspects of the work in ensuring that questions related to the accuracy or integrity of any part of the work are appropriately investigated and resolved. All procedures involved in the animal experiments in this study were performed in line with the National Institutes of Health Guide for the Care and Use of Laboratory Animals. The study was approved by the Ethics Committee of Wuhan 
Third Hospital.

Open Access Statement: This is an Open Access article distributed in accordance with the Creative Commons Attribution-NonCommercial-NoDerivs 4.0 International License (CC BY-NC-ND 4.0), which permits the noncommercial replication and distribution of the article with the strict proviso that no changes or edits are made and the original work is properly cited (including links to both the formal publication through the relevant DOI and the license). See: https://creativecommons.org/licenses/by-nc-nd/4.0/.

\section{References}

1. Cancer Genome Atlas Research N. Comprehensive molecular profiling of lung adenocarcinoma. Nature 2014;511:543-50.

2. Tabbò F, Novello S. Expanding anaplastic lymphoma kinase therapeutic indication to early stage non-small cell lung cancer. Transl Lung Cancer Res 2019;8:S290-7.

3. Gkolfinopoulos S, Mountzios G. Beyond EGFR and ALK: targeting rare mutations in advanced non-small cell lung cancer. Ann Transl Med 2018;6:142.

4. Paez JG, Janne PA, Lee JC, et al. EGFR mutations in lung cancer: correlation with clinical response to gefitinib therapy. Science 2004;304:1497-500.

5. Dehdashti F, Mintun MA, Lewis JS, et al. In vivo assessment of tumor hypoxia in lung cancer with $60 \mathrm{Cu}-$ ATSM. Eur J Nucl Med Mol Imaging 2003;30:844-50.

6. Hockel M, Knoop C, Schlenger K, et al. Intratumoral pO2 predicts survival in advanced cancer of the uterine cervix. Radiother Oncol 1993;26:45-50.

7. Sahlgren C, Gustafsson MV, Jin S, et al. Notch signaling mediates hypoxia-induced tumor cell migration and invasion. Proc Natl Acad Sci U S A 2008;105:6392-7.

8. Schindl M, Schoppmann SF, Samonigg H, et al. Overexpression of hypoxia-inducible factor 1alpha is associated with an unfavorable prognosis in lymph nodepositive breast cancer. Clin Cancer Res 2002;8:1831-7.

9. Zhang L, Wang H, Yang T, et al. Formylpeptide receptor 1 mediates the tumorigenicity of human hepatocellular carcinoma cells. Oncoimmunology 2016;5:e1078055.

10. Le Y, Oppenheim JJ, Wang JM. Pleiotropic roles of formyl peptide receptors. Cytokine Growth Factor Rev 2001;12:91-105.

11. Oldekamp S, Pscheidl S, Kress E, et al. Lack of formyl peptide receptor 1 and 2 leads to more severe inflammation and higher mortality in mice with of pneumococcal meningitis. Immunology 2014;143:447-61.

12. Kamal AM, Hayhoe RP, Paramasivam A, et al. Antiflammin-2 activates the human formyl-peptide receptor like 1. ScientificWorldJournal 2006;6:1375-84.

13. Giebeler A, Streetz KL, Soehnlein O, et al. Deficiency of formyl peptide receptor 1 and 2 is associated with increased inflammation and enhanced liver injury after LPS-stimulation. PLoS One 2014;9:e100522.

14. Zhou Y, Bian X, Le Y, et al. Formylpeptide receptor FPR and the rapid growth of malignant human gliomas. J Natl Cancer Inst 2005;97:823-35.

15. Huang J, Hu J, Bian X, et al. Transactivation of the epidermal growth factor receptor by formylpeptide receptor exacerbates the malignant behavior of human glioblastoma cells. Cancer Res 2007;67:5906-13.

16. Ragone C, Minopoli M, Ingangi V, et al. Targeting the cross-talk between Urokinase receptor and Formyl peptide receptor type 1 to prevent invasion and trans-endothelial migration of melanoma cells. J Exp Clin Cancer Res 2017;36:180.

17. Coffelt SB, Tomchuck SL, Zwezdaryk KJ, et al. Leucine leucine-37 uses formyl peptide receptor-like 1 to activate signal transduction pathways, stimulate oncogenic gene expression, and enhance the invasiveness of ovarian cancer cells. Mol Cancer Res 2009;7:907-15.

18. Snapkov I, Oqvist CO, Figenschau Y, et al. The role of formyl peptide receptor 1 (FPR1) in neuroblastoma tumorigenesis. BMC Cancer 2016;16:490.

19. Cai Y, Huang J, Xing H, et al. Contribution of FPR and TLR9 to hypoxia-induced chemoresistance of ovarian cancer cells. Onco Targets Ther 2019;12:291-301.

20. Cheng TY, Wu MS, Lin JT, et al. Formyl Peptide receptor 1 expression is associated with tumor progression and survival in gastric cancer. Anticancer Res 2014;34:2223-9.

21. Prevete N, Liotti F, Visciano C, et al. The formyl peptide receptor 1 exerts a tumor suppressor function in human gastric cancer by inhibiting angiogenesis. Oncogene 2015;34:3826-38.

22. Cairns RA, Kalliomaki T, Hill RP. Acute (cyclic) hypoxia enhances spontaneous metastasis of KHT murine tumors. Cancer Res 2001;61:8903-8.

23. Shi L, Wang Z, Liu X, et al. Bax inhibitor-1 is required for resisting the Early Brain Injury induced by subarachnoid hemorrhage through regulating IRE1-JNK pathway. Neurol Res 2018;40:189-96.

24. Livak KJ, Schmittgen TD. Analysis of relative gene expression data using real-time quantitative PCR and the 2(-Delta Delta C(T)) Method. Methods 2001;25:402-8. 


\section{Page 10 of 10}

25. Weidemann A, Johnson RS. Biology of HIF-1alpha. Cell Death Differ 2008;15:621-7.

26. Liu C, Shui CL, Wang Q, et al. Mechanism of hif-1alpha mediated hypoxia-induced permeability changes in bladder endothelial cells. Braz J Med Biol Res 2017;51:e6768.

27. Brahimi-Horn MC, Chiche J, Pouyssegur J. Hypoxia and cancer. J Mol Med (Berl) 2007;85:1301-7.

28. Gilkes DM, Semenza GL, Wirtz D. Hypoxia and the extracellular matrix: drivers of tumour metastasis. Nat Rev Cancer 2014;14:430-9.

29. Silva P, Mendoza P, Rivas S, et al. Hypoxia promotes Rab5

Cite this article as: Huang B, Guo H, Ding J, Li J, Wang H, Xu J, Zheng Q, Zhou L, Dai Q. Inhibition of formyl peptide receptor 1 activity suppresses tumorigenicity in vivo and attenuates the invasion and migration of lung adenocarcinoma cells under hypoxic conditions in vitro. Ann Transl Med 2020;8(18):1174. doi: 10.21037/atm-20-5864

\section{Huang et al. FPR1 involves in tumor growth and invasion}

activation, leading to tumor cell migration, invasion and metastasis. Oncotarget 2016;7:29548-62.

30. Sullivan R, Graham CH. Hypoxia-driven selection of the metastatic phenotype. Cancer Metastasis Rev 2007;26:319-31.

31. Vaupel P. Hypoxia and aggressive tumor phenotype: implications for therapy and prognosis. Oncologist 2008;13 Suppl 3:21-6.

32. Xue X, Chen XX, Wang G, et al. Formyl peptide receptor 1 promotes migration in mouse microglial cell line BV-2. Journal of Third Military Medical University 2016;1:44-9. 